Revue internationale P.M.E.

Économie et gestion de la petite et moyenne entreprise

\title{
La PME innovatrice : Quel est le rôle du milieu local ?
}

\section{Bernard Planque}

Volume 1, numéro 2, 1988

URI : https://id.erudit.org/iderudit/1007880ar

DOI : https://doi.org/10.7202/1007880ar

Aller au sommaire du numéro

Éditeur(s)

Presses de l’Université du Québec

ISSN

0776-5436 (imprimé)

1918-9699 (numérique)

Découvrir la revue

Citer cet article

Planque, B. (1988). La PME innovatrice : Quel est le rôle du milieu local ? Revue internationale P.M.E., 1(2), 177-191. https://doi.org/10.7202/1007880ar

\section{Résumé de l'article}

Dans un effort pour mieux comprendre quelle peut être l'incidence d'un milieu local sur les capacités d'innovation réussie des PME, nous présenterons brièvement, en première partie, une caractérisation globale des processus d'innovation dans leurs composantes multifonctionnelles, multisectorielles, multilocales et dynamiques. Puis, en deuxième partie, une proposition de caractérisation des comportements de recherche d'informations et de compétences par les PME innovatrices. La mise en relation des deux types de caractérisation permet, en conclusion, d'avancer l'hypothèse selon laquelle le potentiel territorial d'information et de compétences module, du moins en partie, les probabilités d'émergence et de conduite à terme des processus d'innovation dans les PME. 


\title{
La PME innovatrice : Quel est le rôle du milieu local ?
}

\author{
Bernard PLANQUE \\ Centre d'économie régionale \\ Université d'Aix-Marseille III
}

\begin{abstract}
RÉSUME
Dans un effort pour mieux comprendre quelle peut être l'incidence d'un milieu local sur les capacités d'innovation réussie des PME, nous présenterons brièvement, en première partie, une caractérisation globale des processus d'innovation dans leurs composantes multifonctionnelles, multisectorielles, multilocales et dynamiques. Puis, en deuxième partie, une proposition de caractérisation des comportements de recherche d'informations et de compétences par les PME innovatrices. La mise en relation des deux types de caractérisation permet, en conclusion, d'avancer l'hypothèse selon laquelle le potentiel territorial d'information et de compétences module, du moins en partie, les probabilités d'émergence et de conduite à terme des processus d'innovation dans les PME.
\end{abstract}

\begin{abstract}
For a better understanding of what could be the effect of the local milieu surrounding the small firms on its successful innovations, firstly, we will explain some global characteristics of the innovative process in their multifunctionnal, multisectorial, multilocal and dynamical parts. Secondly, we will present a proposal for the characterisation of the small firms innovator behaviours in the research for information and competencies. Finally, the links between these two types of characteristics will allow us to introduce the hypothesis that the local informational and competency possibilities will determine a great part of the probabilities for emerging and acting the innovative process in small firms on the long term.
\end{abstract}

\section{RESUMEN}

Para esforzarnos de comprender mejor cual puede ser la incidencia de un medio local sobre las capacidades de innovacion aseguradoras de éxito de la PyME presentaremos brevemente una caracterizacion global de los procesos de innovacion en sus composantes multifoncionales, multisectoriales, multilocales y dinamicas (1era parte) y una proposicion de caracterizacion de los comportamientos de busqueda de informacion y de competencias por parte de las PyME innovadoras (2da parte). Relacionar los dos tipos de caracterizacion permite en conclusion avanzar la hipotesis segun la cual, el potencial territorial de informacion y de competencias modula, al menos en parte, las probabilidades de emergencia y de conduccion al termino, de los procesos de innovacion en las PyME. 


\section{Quelques caractéristiques des processus d'innovation}

Divers travaux antérieurs du Centre d'économie régionale (Granie, 1985; Planque, 1981, 1983; Perrin, 1985) nous ont conduits à concevoir l'innovation réussie comme résultant d'un processus de coproduction fondamentalement collectif, multifonctionnel, multisectoriel, multilocal et inscrit dans le temps. Dans de tels processus, les articulations interorganisationnelles entre «partenaires» impliqués à un titre ou à un autre jouent, selon toute vraisemblance, un rôle central.

L'innovation suppose la collaboration et l'enchainement réussi de fonctions et d'étapes complémentaires: recherche fondamentale, recherche appliquée, développement, élaboration de prototypes, investissement industriel, mise en production, commercialisation, adaptation de la production au marché... Chacune de ces fonctions de l'organisation exigeant une combinaison spécifique d'intrants au sens le plus large : de compétences, de qualifications du travail, d'informations, de capital physique et financier.

Pendant le déroulement du processus d'innovation, l'importance relative des fonctions, leur mode d'articulation entre elles et avec l'environnement, donc leurs combinaisons d'intrants, tendent à se modifier.

Cette différenciation des combinaisons d'intrants selon les étapes se traduit à la fois par une différenciation des modes internes d'organisation fonctionnelle (au sens de l'entreprise) et par une différenciation de la structure des appels à l'extérieur, des modes de relations ennvironnementales.

Le caractère multifonctionnel des processus d'innovation se double souvent d'un caractère multisectoriel. Une grande partie des innovations découle de l'adaptation hors de son secteur d'origine d'une méthode, d'une technique, d'un concept et du mariage entre des méthodes, des techniques, des concepts et des procédés qui ne s'étaient pas rencontrés jusque là. Nous verrons que ce caractère multisectoriel (et multidisciplinaire) de l'innovation prend aujourd'hui une importance particulièrement grande dans le domaine des activités qu'il est convenu d'appeler «activités à haute technologie» (cf. période 1-2). Les compétences multisectorielles sont rares, surtout dans les PME où les collaborations multisectorielles sont rendues difficiles par la méconnaissance réciproque, le caractère informel et l'origine souvent contingente des systèmes de contacts interindividuels ou interorganisationnels.

Nous pouvons caractériser l'innovation réussie comme résultant d'un processus collectif de coproduction. Même s'il est vrai que l'idée d'innovation, l'effort principal d'innovation et la réalisation concrète de la nouvelle combinaison sont le fait de l'unité microéconomique et, souvent dans les PME, d'un ou de quelques individus; la probabilité d'émergence de l'idée nouvelle et la probabilité de réussite du processus d'innovation dans son ensemble dépendent fortement du milieu dans lequel cette organisation microéconomique se trouve plongée ainsi que 
de son mode d'insertion dans ce milieu. Par «milieu» il faut entendre le système de l'ensemble des éléments interreliés capables de fournir au candidat innovateur l'ensemble des intrants différenciés utiles à l'ensemble des étapes dont se compose son processus innovateur. Le caractère privilégié ou plus apparent de certains éléments de ce milieu et de certaines relations (telles les relations «rechercheindustrie» par exemple) ne doit pas masquer le fait que ce milieu est un système dont tous les sous-systèmes participent au fonctionnement de l'ensemble. Notre analyse du «technopole» Silicon Valley a permis de montrer comment ce n'est pas seulement la qualité des recherches de l'université Stanford et ses bonnes relations avec les entreprises de «haute technologie» locales qui explique l'extraordinaire dynamique innovatrice qu'a connue cette région. Bien d'autres éléments participent à cette explication : accès privilégiéà des formes spécifiques de financementet d'information financière, présence locale d'un marché public porteur, services spécialisés dans plusieurs domaines clef, sous-traitants spécialisés... (Planque, 1985).

La spécialisation des compétences dont la combinaison doit être assurée pour conduire à terme un projet innovateur nous conduit à émettre l'hypothèse selon laquelle, sur un plan local ou régional, la proximité entre partenaires facilitant les contacts interpersonnels directs, l'organisation des réseaux relationnels (locaux, régionaux) peut être un facteur non négligeable de stimulation ou de freinage des capacités d'innovation. Cependant le double caractère multifonctionnel et multisectoriel des processus d'innovation conduit habituellement à un éclatement dans l'espace (interrégional, international) des ressources utiles à leur réalisation («intrants»). En ce sens, les processus d'innovation possèdent un caractère multilocal (multirégional, multinational) et demandent l'utilisation de réseaux interlocaux de circulation des informations.

Pour résumer, l'innovation, la réalisation d'une «combinaison nouvelle», suppose la capacité de faire converger des éléments, des informations, des flux, des compétences d'origine toujours multifonctionnelle, souvent multisectorielle, presque toujours multilocale.

Les PME doivent, pour réussir une innovation, trouver les moyens de faire converger vers elles, pour les combiner, despotentialités techniques, informationnelles, humaines, financières éclatées tant sur les plans sectoriels et fonctionnels que sur le plan spatial, sans disposer des structures qui permettent aux grandes firmes d'effectuer systématiquement ces opérations.

A ces caractéristiques de multifonctionnalité, de multisectorialité et de multiterritorialité des processus innovateurs, il convient de superposer leur caractéristique de dynamisme. Ces processus sont inscrits dans le temps; la réussite d'un programme innovateur ne se résume pas à la réalisation d'une nouvelle combinaison ou d'un nouveau produit; elle inclut les étapes décisives de l'industrialisation et de la commercialisation, ce qui suppose le dépassement du problème toujours central du financement des investissements. Ainsi la réussite finale d'un programme innovateur ne signifie pas seulement la capacité de faire converger des potentialités et des ressources utiles à un moment donné (par exemple 
des ressources informationnelles scientifiques et techniques lors des étapes initiales du processus); elle signifie aussi la capacité de faire évoluer l'organisation (interne de l'entreprise) et les sources (externes) d'intrants en fonction des nouvelles combinaisons de facteurs (informationnels, humains, matériels) qui se différencient d'étape en étape (ce point est développé sur le plan théorique dans Planque et Py, 1987 et dans une étude détaillée de cas de Planque, Py, Proulx et Radjama, 1987).

\section{PME innovatrices et recherche à courte séquence des informations et des compétences}

L'information (les informations de types et de qualités différenciés) est un facteur de production dont il est clair qu'il joue un rôle essentiel dans la vie économique, tout particulièrement en matière d'innovation même si nous ne savons correctement en mesurer ni le coût ni la productivité.

Cerôle essentiel tient notamment au fait que l'accessibilitéà des informations à propos de l'environnement, permet de réduire l'incertitude et par là, d'adopter des stratégies appropriées (Lambooy, 1986).

L'information s'obtient par la communication. Cette communication a un coût variable selon les types d'information qu'elle véhicule et selon les canaux qu'elle emprunte, selon l'organisation des réseaux communicationnels. Reprenant la terminologie qu'utilisent certains linguistes (Descles, 1980) nous pouvons schématiquement opposer un type de communication «monologique» à un type de communication «dialogique» (Planque, 1983). La communication qualifiée de «monologique» est celle qui réduit au minimum «le problème de l'ajustement intersubjectif entre l'énonciateur qui produit un texte et son co-énonciateur qui le comprend» (Descles, 1980) et celle qui a codé l'information de façon non ambiguë; elle suppose une compréhension immédiate du signe transmis, sans nécessiter de réponse ou d'ajustement progressif. Ce type de communication concernel'information formellement codée (texte écrit, données chiffrées, ordres et questions précises) qui présente la caractéristique importante d'être accessible (au moins potentiellement) depuis un lieu quelconque avec un coût qui ne varie que marginalement selon la distance entre l'origine et le point d'arrivée de l'information. En d'autres termes, il s'agit d'un type d'information aisément télétransmissible et ce, sans que la distance ait beaucoup d'incidence sur le coût de l'obtention de l'information (ce qui est presque réussi avec l'amélioration spectaculaire des techniques de télécommunication). 
Par opposition à ce type de communication, nous pouvons qualifier de «dialogique» la transmission d'informations comportant un risque élevé de malentendus, nécessitant des aller-retour et dont la formalisation a priori est difficile. Cette communication dialogique caractérise notamment les procédures de négociation.

La contractualisation et l'institutionnalisation (H. Simon) sont des moyens par lesquels les organisations cherchent à réduire l'incertitude et par là à réduire les coûts de transaction attachés aux types d'information communiqués sur ce mode dialogique.

Les grandes entreprises, s'appuyant sur leur structuration formelle et sur la spécialisation des individus, des équipes, des services, se procurent les divers types d'informations utiles à leurs processus de production et d'innovation au travers de réseaux institutionnels. Elles recherchent explicitement et de façon systématique les informations et, le plus souvent, s'efforcent de réduire l'incertitude quant à leur environnement en contractualisant leurs relations avec les éléments, les «partenaires» de cet environnement.

Une spécificité importante des petites et moyennes entreprises par rapport aux grandes organisations apparaît dans leur inaptitude à l'institutionnalisation (impossibilité de structuration par spécialisation) et dans leur pratique qui consiste à ne pas contractualiser systématiquement leurs relations. Cette caractéristique des PME, d'autant plus affirmée que l'entreprise est plus petite et/ou plus jeune, se traduit par une conformation originale (par rapport aux grandes organisations) des réseaux de mobilisation des intrants informationnels.

Quelques exemples tirés notamment d'une étude de cas approfondie de la dynamique de l'insertion environnementale d'une petite entreprise innovatrice permettent de préciser notre propos (Le cas S dans Planque, Py, Proulx et Radjama, 1987).

Le réseau de mobilisation d'information le plus «simple« que nous ayons rencontré lors de ce cas $\mathrm{S}$ se situe dans une étape antérieure à la naissance de l'entreprise elle-même, au moment où sa gestation mobilise toute l'énergie de celui qui deviendra son créateur (PV).

Ace stade de l'affaire, on peut représenter l' «entreprise» comme un individu recherchant activement un «intrant» particulier, une information technique : existet-il un produit nouveau dont il ait les moyens d'assurer la production et la commercialisation? Les sources d'information (l'origine des intrants) sont les multiples acteurs intervenant à un titre ou à un autre dans le «milieu» sectoriel choisi : ici dans le cas $S$, celui de la navigation de plaisance. Le vecteur d'obtention de l'information (ou le type de communication) est un ensemble de relations interpersonnelles informelles, non institutionnalisées et non structurées. La localisation des «noeuds» du réseau, étant donné le type de communication utilisé est principalement limitée à la région dans laquelle l'innovateur potentiel est susceptible de se déplacer aisément. 
Schématiquement, àce degré d'incubation de l'entreprise on peut représenter son insertion environnementale :

\section{Fig. 1. Insertion environnementale de l'embryon d'entreprise pour la période $1-1$}

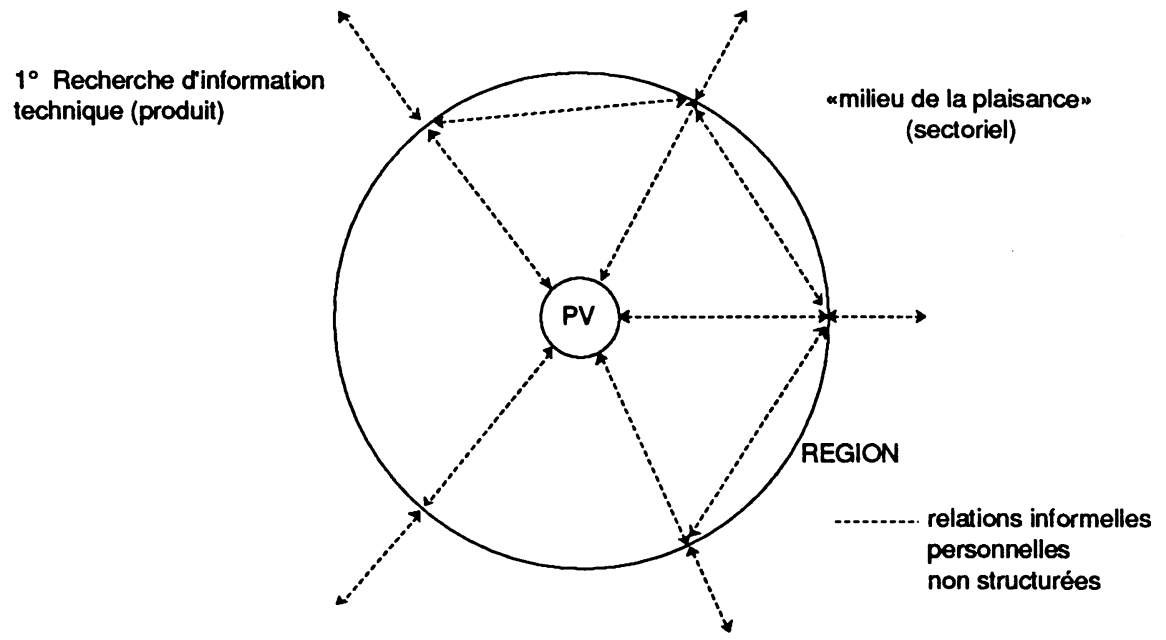

Au cours de ce tout premier stade, l'«entreprise» n'étant encore que virtuelle, on observe, caricaturée à l'extrême, ce qui est peut-être l'une des caractéristiques les plus marquantes des petites entreprises : l'immersion dans un «milieu» (à la fois professionnel, sectoriel et local) porteur, émetteur d'informations dont elles s'efforcent de happer les bribes qui se présentent à elles.

Certes, dès que l'entreprise, perdant son caractère virtuel deviendra réelle, le réseau dans lequel elle s'insère va se complexifier, se démultiplier, faire coexister et se superposer de multiples sous-réseaux sur les divers plans fonctionnels (technique, financier, commercial, pour l'essentiel); mais ces divers sous-réseaux, même s'ils s'enrichissent au cours du développement de l'entreprise de dimensions supplémentaires, de types différents de communication, resteront marqués jusqu'à un niveau très élaboré de structuration de l'organisation par des relations interpersonnelles directes entre responsables internes et émetteurs potentiels d'informations externes.

Ce type de mode de communication est directement lié à une caractéristique de comportement très générale dans les petites organisations : elles ne cherchent pas 
vraiment à disposer d'une information complète à la fois parce que le coût d'une telle information est prohibitif pour elles et parce qu'elles n'auraient, en toute hypothèse, pas les moyens de traiter, de trier une telle information.

Qu'il s'agisse du plan technique, du plan commercial ou du plan financier, il est clair dans le cas que nous avons analysé que l'acteur économique est incapable d'avoir une information synoptique globale et que, selon la formule de Crozier et Frieberg (1977) : «il décide de façon séquentielle et choisit pour chaque problème qu'il a à résoudre la première solution qui correspond pour lui à un seuil minimal de satisfaction». Au cours du développement et de la structuration ultérieure de l'entreprise, les «séquences» pourronts'allonger quelque peu, la quête de l'information devenir un peu plus systématique, tout en restant toujours partielle et très éloignée de «l'information parfaite» dont la Science économique postule trop souvent que les agentséconomiques sont dotés; quand un acteur pense avoir «l'information minimale suffisante», il décide et agit.

Pour rester sur le plan des relations d'information technique, à un niveau un peu plus avancé du développement de l'entreprise, il n'est pas sans intérêt d'observer le réseau tel qu'il se met en place lorsque notre petite entreprise (désormais créée et en cours d'installation physique) va se trouver contrainte de développer une innovation de procédé.

La jeune entreprise du cas $S$ a développé un produit nouveau, un type particulier, inconnu jusque-là, de mât de planche à voile à hautes performances, fabriqué dans un «matériau nouveau», un alliage léger jusque-là exclusivement utilisé en aéronautique et dont le traitement thermique s'avère particulièrement délicat.

Dans un premier temps, $\mathrm{S}$ envisage de faire sous-traiter cette étape des «traitements thermiques complexes». Quatre sociétés de la région équipées de fours capables de réaliser cette opération sont contactées (il s'agit de fours verticaux avec plongées des charges en piscine). Aucune d'elles ne peut traiter plus de 500 à 1000 pièces, de plus la stabilité de la qualité ne semble pas assurée. Seule, la SNIAS serait disposée à réaliser cette opération dans ses propres fours mais sans pouvoir assurer à $S$ une production régulière. De ces difficultés pour trouver une sous-traitance adaptée à ces nouveaux besoins va naître une innovation de procédé.

$S$ cherche à acheter un four adapté à ses besoins, s'informe auprès des fabricants français, puis allemands (première tentative d'ouverture internationale du réseau), aucun équipement adapté ne semble exister sur le marché.

Ayant glané des informations auprès de relations des ingénieurs à la SNIAS (canal d'information informelle, non contractuelle, déjà utilisée lors de la mise au point du nouveau produit «mât»), de spécialistes amis travaillant chez Péchiney et de fabricants de fours industriels, PV va concevoir et construire son propre four «horizontal», d'une capacité de 90000 mâts par an, installé dans l'usine elle-même (ce qui permettra l'automatisation de la chaîne de production et réduira les coûts de manutention et de transport). Les sous-ensembles mécaniques nécessaires à la 
construction du four seront fabriqués à la pièce par une chaudronnerie locale, habituellement sous-traitante des chantiers navals de La Seyne (S est installée à Six Fours, tout près de la Seyne).

Tout comme dans le cas de l'étude d'un nouveau matériau et de ses modalités de traitement, on observe ici des transferts de technologie involontaires et non contractuels entre de grandes entreprises (SNIAS, Péchiney, fabricants de fours, Chantiers Navals) et la PME, par l'intermédiaire de cadres des grandes entreprises et/ou de leurs sous-traitants. Là encore, point d'information synoptique; le responsable de la petite entreprise consulte des connaissances diverses, rencontrées au hasard des relations personnelles, plus quelques spécialistes (les fournisseurs potentiels). Très rapidement, il passe à l'action (cf. fig. 2).

Fig. 2. Réseau d'accès à l'information technique pour innovation-procédé «pour industriel» pendant l'étape 3

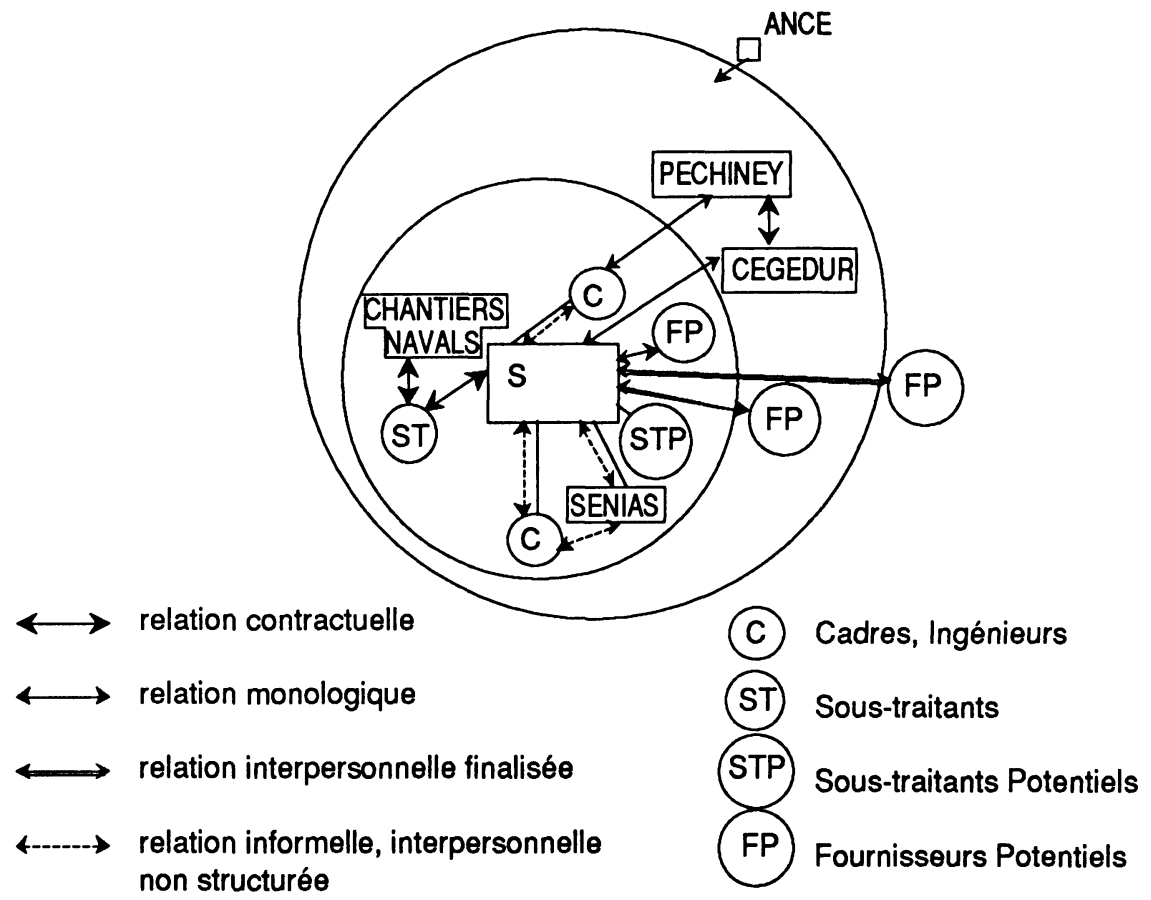

Il serait possible, même à partir de cette seule étude de cas, de multiplier les exemples en examinant l'organisation, aux différentes étapes du développement de l'entreprise, des réseaux sur les plans commerciaux et financiers. L'objet de cet article n'est pas de restituer l'étude du cas S diffusée par ailleurs par Planque et alii 
(1987) mais de tenter de mieux comprendre comment les caractéristiques de l'environnement territorial d'une PME innovatrice influencent sa capacité (ou sa probabilité) de réussite. Il est clair que, du point de vue de l'information technique quiétaitrecherchée dans l'exempleci-dessus, si lescanaux d'obtention del'information sont bien du type que nous avons rapidement décrit, plus le milieu territorial (la région dans laquelle se noue l'essentiel des relations personnelles) est riche en émetteurs potentiels d'information adaptée et plus la vraisemblance pour la PME de rencontrer l'information correcte (celle qui permettra la mise en place d'une technologie appropriée) plus cette vraisemblance est grande. Le même type d'argument peut naturellement être avancé pour l'information commerciale ou pour l'information sur les moyens de financement et pour celui de l'obtention de ces moyens.

L'élément central qui nous paraît caractériser les premières étapes de la mise en place d'une PME innovatrice réside dans la relative efficacité d'une quasi absence de structuration explicite tant du point de vue organisationnel (interne) que du point de vue relationnel (externe).

a) Sur le plan organisationnel, même si des éléments de spécialisation des tâches se sont progressivement mis en place, une certaine polyvalence des individus reste de règle. Le chef d'entreprise est toutà la fois technicien, concepteur, ingénieur, chercheur, relationniste, agent commercial, gestionnaire, réparateur, acheteur, contrôleur de production, livreur à l'occasion... Les premiers ouvriers embauchés sont manutentionnaires, livreurs, participent à la construction de l'outil de production, à son entretien autant qu'à la production elle-même. Le «directeur commercial» est vendeur, assistant technique des supports publicitaires («sponsoring»), relationniste, responsable des exportations, ... sans que le partage des rôles, des tâches et des responsabilités avec le chef d'entreprise ne soit jamais très clairement explicité. Les deux secrétaires sont à la fois dactylographes, comptables, standardistes, facturières, prennent les commandes, réceptionnent produits, clients, fournisseurs...

Cette polyvalence fonctionnelle des individus, si elle entraîne certainement comme conséquence (par rapport à une organisation à forte spécialisation des tâches) une moindre efficacité de chacune des fonctions de l'entreprise considérée isolément, conduit aussi, pour une très petite organisation constituée par quelques individus capables de dialogue direct et permanent, à une excellente intégration interfonctionnelle. Cette articulation des fonctions entre elles, non strictement formalisée, non inscrite sur un organigramme institutionnel mais réalisée par synthèse individuelle ou interindividuelle est probablementl'une des sources importantes de cette souplesse, de cette capacité d'adaptation que l'on se plaîtà reconnaître aux petites organisations. Dans le même temps, la faible spécialisation des tâches constitue (avec d'autres éléments tel que le choix de matériels, de produits, de marchés... effectués en contexte d'information très partielle) une très forte source de fragilité pour une petite organisation située dans un marché concurrentiel. L'innovation, le lancement d'un produit nouveau en mettant provisoirement et partiellement l'entreprise à l'abri de la concurrence, lui permet de supporter - au moins pour un temps - le fait de fonctionner avec une combinaison productive très éloignée de l'optimum possible. 
b) Du point de vue relationnel (entreprise/environnement) nous avons souligné à de nombreuses reprises sur les divers plans fonctionnels observés, le caractère partiel peu systématique, non synoptique de la recherche d'information. L'importance des relations interpersonnelles non contractuelles est apparue dans la plupart des domaines, de même que le caractère très approximatif des tentatives de contractualisation

Ce type de connections environnementales, s'il a naturellement pour inconvénient de laisser l'entreprise dans une grande incertitude objective quant à l'état de son environnement, a pour avantage de lui fournir à très faible coût une information, certes très partielle, mais suffisante étant donné le contexte: l'entreprise est très petite, le marché en forte croissance, le produit quasiment sans concurrence (sur le créneau haut de gamme) et la marge sur l'unité vendue est considérable. Dans ces conditions - liées simultanément au fait de la petite taille et au caractère effectivement innovateur de son produit - des erreurs d'appréciation, dues à une information très imparfaite, qui seraient sans doute fatales dans d'autres contextes restent supportables. Par exemple, dans le cas de $S$ une erreur d'estimation de $40 \%$ sur les quantités produites et vendues en 1982 (12000 mâts au lieu de 20000 prévus) s'est soldée par ... un bénéfice qui représente $1 / 3$ du chiffre d'affaires et qui couvrait en un an l'intégralité des investissements réalisés.

Dans le même ordre d'idées, la politique - on pourrait dire l'absence de politique - d'embauche est un exemple d'action appuyée sur une information séquentielle (à très courte séquence) sans recherche synoptique : le fondateur de l'entreprise, trop pris par ses multiples préoccupations pour avoir les moyens (principalement le temps) de rechercher le spécialiste ad hoc pour telle ou telle fonction, lié par diverses contraintes, embauche tel petit actionnaire pour assurer la direction commerciale, tel ami d'un ami, comme chef d'équipe, sa propre épouse, la fiancée d'une relation comme secrétaire... La relation de confiance personnelle ${ }^{1}$ domine sur la relation contractuelle. Et tant que l'équipe est très petite, ce type de relation ne semble pas manquer d'une certaine efficacité.

Cependant et très rapidement, si l'innovation parvient à s'imposer sur le marché, le changement de taille de l'organisation, obligé par le changement d'échelle de production, va entraîner des tentatives de structuration interne et de maîtrise plus systématique que par le passé des relations avec l'environnement. Ces efforts de structuration, réels et entrepris explicitement, vont, au moins dans un premier temps, être conduits - en quelque sorte paradoxalement - en s'appuyant sur les réseaux non formalisés et non explicités qui ont été mis en place au cours des étapes antérieures. La petite entreprise en croissance va ainsi s'efforcer de bâtir une organisation formelle, un organigramme interne et une institutionnalisation des relations externes à partir de ses réseaux peu formels anciens.

1 Cette relation de confiance, non seulement à l'intérieur de l'entreprise mais aussi dans les relations interpersonnelles externes, est assimilée par De Bernardy et Boisgontier (1987) à «un bien capital» dans le contexte risqué de l'innovation pour des PME. 
Analysant a posteriori le cas $\mathrm{S}$, et ayant dans le même temps des relations suivies avec d'autres petites unités innovatrices (et jeunes), il nous semble que cette contradiction entre, d'une part, la volonté et la nécessité ressentie de diriger l'organisation vers un modèle formellement structuré (aux sens interne et externe) lorsque sa taille s'accroît et, d'autre part, les réflexes d'action en milieu peu structuré formellement hérités des premières étapes de développement, constitue l'une des difficultés majeures, l'un des facteurs principaux de risque de crise grave que rencontrent ces entreprises dans la transition entre la petite unité et l'entreprise moyenne ${ }^{2}$.

La démarche de recrutement, d'un nouveau directeur commercial par exemple, ne manque pas d'intérêt et souligne la contradiction centrale dans laquelle se trouve la PME quand sa taille la pousse à la structuration interne par spécialisation des tâches pour améliorer sa maîtrise environnementale. L'objectif de l'embauche d'un expert en matière commerciale est clairement de tenter de réduire les coûts de transaction dans le domaine particulier des relations avec les clients. Embaucher un expert pour lui confier la responsabilité d'un plan fonctionnel particulier est typiquement une action de structuration et d'institutionnalisation telle que la pratique couramment une grande organisation. Pourtant, la démarche d'embauche reste typique d'une petite organisation insuffisamment structurée pour chercher à obtenir explicitement une information tant soit peu synoptique pour éclairer son choix. Dans le cas particulier, PV entretient des relations amicales avec YP, actionnaire de S. YP est lui-même lié depuis longtemps à un responsable commercial BL d'une grande entreprise (Brandt), lequel responsable commercial est réputé excellent vendeur. BL, stimulé par les perspectives de croissance de $S$, par une rémunération plus élevée et par l'autonomie totale d'organisation et de direction de ses activités commerciales qui lui est proposée, devient donc directeur commercial de l'entreprise sans même qu'aucune autre candidature n'ait été envisagée. Ainsi pourrions-nous avancer qu' un effet important d'un milieu local sur une PME en voie de structuration tient probablement à la richesse relative de son potentiel de compétences. Ce type d'entreprise - on y a déjà insisté - n'a pas les moyens (moins en termes financiers qu'en termes culturels) d'envisager une recherche systématique des compétences ad hoc et elles sont conduites à choisir dans le pannel qui se présente à elles, souvent au travers des réseaux de relations personnelles. En ce sens, un milieu local riche en compétences adaptées aux problèmes de la PME en voie de structuration doit tendre à augmenter la probabilité que, sans les avoir réellement recherchées, l'entreprise se dote descompétences appropriées. Inversement, un milieu local pauvre en compétences adaptées pourrait tendre à diminuer cette probabilité. Dans le cas de $S$, le milieu local (région de Toulon/La Seyne) est riche en compétences techniques (niveau ouvrier qualifié/technicien) disponibles (notamment du fait des compressions d'effectifs

2 Notons au passage que le «seuil» d'une dizaine de salariés généralement considéré comme significatif pour distinguer les entreprises «petites» des «moyennes» pourrait correspondre à un seuil organisationnel/relationnel, celui au-delà duquel la quasi absence de structuration explicite cesse d'être efficace. 
dans les chantiers navals et de leurs conséquences chez les sous-traitants) mais pauvre dans d'autres types de compétences. En conséquence, excepté pour les recrutements de techniciens et des ouvriers, le pannel de compétences qui s'offrent spontanément (sans recherche, sans avoir à supporter les coûts d'une recherche d'information) est très limité et la probabilité pour l'entreprise de «tomber sur» la compétence adaptée est faible. Dans certaines régions du monde, notamment celles où se sont développées des activités «à haute technologie» particulièrement avides de compétences pointues (cf. section 1), ce sont non seulement des réseaux locaux d'information sur les compétences des individus qui se sont développés (cf. Planque, 1985 sur le cas de la Silicon Valley) mais aussi la pratique du recours aux «chasseurs de tête». Ces «chasseurs de tête» jouent un rôle essentiel de réducteurs del'incertitude dans la perspective des recrutements tout en opérant, du fait de leur spécialisation, une réduction importante du coût de la recherche de ce type d'information (pour certaines régions des USA, Rogers, 1986; pour la Silicon Valley, Rogers and Larsen, 1984; pour Cambridge, Segal et alii, 1985; de façon générale sur le rôle des «headhunters» dans l'interconnection des réseaux informationnels/relationnels habituellement séparés, MacDonald, 1986).

\section{Conclusion : Le rôle du potentiel territorial d'information et de compétences}

Quelles conclusions peut-on tirer des réflexions présentées ci-dessus du point de vue de la compréhension des phénomènes de «synergies locales» ou de «synergies territoriales» ${ }^{3}$ en matière d'émergence et de réussite globale des processus d'innovation conduits par des PME?

- Si, comme nous l'avons souligné dans la section 1 , la réussite globale d'un programme innovateur suppose la capacité de faire converger sur l'organisation innovatrice des combinaisons d'intrants (notamment informationnels et de compétences) d'origines très diverses et des combinaisons qui tendent à se modifier d'étape en étape au cours du processus d'innovation

- si, comme nous en avons proposé l'hypothèse dans la section 2 , les petites entreprises innovatrices ont tendance pour des raisons multiplesà s'approvisionner en intrants (notamment informationnels et de compétences) selon des procédures séquentielles très courtes, en happant les informations et les compétences qui se présentent à elles et leur évitent le coût d'une recherche synoptique,

3 Concepts largement discutés dans la littérature récente : Perin, 1987; Stohr, 1986; Andersson, 1985 et Planque (éd.), 1987. 
- si la proximité est un facteur favorable aux rencontres interpersonnelles par lesquelles sont véhiculées une grande partie de ces informationset si la proximité est un facteur favorable à la liaison de relations de confiance qui donnent, du point de vue de la petite entreprise, leur fiabilité à ces informations,

- alors, nous pouvons probablement admettre que les structures locales jouent un rôle d' «institution informelle» : "dans les systèmes socio-économiques, les institutions sont une trame instrumentale grâce à laquelle plus de continuité, de stabilité et de cohérence sont introduites dans les relations humaines» (Perrin, 1987).

Nous sommes ainsi conduits à avancer qu'un milieu local est d'autant plus favorable à l'émergence et à la réalisation réussie dans son ensemble de processus innovateurs conduits dans des PME, qu'il maximise la probabilité pour ces PME d'obtenir sans coût (ou à faible coût, i.e. au terme d'une brève séquence de recherche non synoptique d'information) les informations et/ou les compétences appropriées étant donné les problèmes fonctionnellement différenciés qui se posent à elles au cours des différentes étapes de leurs processus innovateurs. Nous sommes conduits à conclure qu'un milieu local est d'autant plus favorable, selon cette perspective, qu'il dispose d'un potentiel informationnel élevé dans les divers domaines utiles à l'innovation et qu'il dispose d'un potentiel élevé de compétences ou de «savoirfaire» accumulé selon l'expression de Maillat (1987). Une condition supplémentaire pour que l'institution informelle fonctionne efficacement doit être soulignée : encore faut-il que le potentiel informationnel local soit accessible aux PME.

L'accessibilité du potentiel informationnel local pour les PME dépend probablement de facteurs multiples parmi lesquels on peut distinguer deux groupes complémentaires : d'une part des facteurs de type organisationnel, d'autre part des facteurs d'ordre culturel.

Les facteurs de type organisationnel tiennent en grande partie à l'existence ou à l'absence, selon les territoires, de structures qui peuvent prendre des formes très variables, capables d'apporter une information triée, «prédigérée» aux PME innovatrices et ceci non seulement sur le plan technique mais aussi sur les autres plans fonctionnels. Lorsqu'on analyse par exemple le développement de la Silicon Valley, le rôle éminent qu'y ont joué les entreprises de «venture capital» par leur insertion dansles «start-up», leurstigoureux conseils de gestion, d'organisation, de management, de marketing ... est tout à fait frappant (Planque, 1985). Dans de nombreux autres cas, ce sont des structures d' incubation, des «centres d'entreprises» (Merenne-Schoumaker, 1987), des «incubateurs» divers qui s'efforcent d'apporter aux PME innovatrices des informations pré-traitées. Dans de nombreux territoires, aucune structure n'assure cette fonction et, très vraisemblablement, les difficultés pour mener à bien un programme innovateur s'en trouvent considérablement accrues, leur probabilité de réussite s'en trouve amoindrie.

Les facteurs de type culturel, pour être moins apparents, n'en sont pas moins importants. Dans le cas de la Silicon Valley, il est clair que la communauté de 
formation initiale et permanente dans les mêmes unités d'une part et l'intense mobilité des hommes entre les entreprises et leurs partenaires d'autre part ont été un facteur puissant de communauté culturelle, de connaissance réciproque des individus et, par là, un facteur décisif de circulation des informations, une garantie d'accessibilité au formidable potentiel informationnel local pour les PMI où travaillent ces individus. Dans un cas moins prestigieux, tel que celui du «Pays d'Aix-en-Provence» où se multiplient rapidement des activités nouvelles, souvent innovatrices, souvent sous forme de petites entreprises, on est frappé de constater qu'il existe entre les responsables de ces unités une forme de communauté culturelle qui n'est pas liée à l'origine locale de ces responsables (dont très peu sont issus de la région) mais à un passé professionnel commun dans un petit nombre de grandes entreprises (Radjama, 1986).

C'est, semble-t-il, en grande partie de ses capacités organisationnelles et de ses capacités culturelles à rendre accessibles son potentiel d'information et de compétences aux PME que dépend la puissance synergétique d'une «institution informelle» territoriale dans le domaine de l'innovation.

\section{BIBLIOGRAPHIE}

Andersson, A.E., (1985), «Creativity and regional development», Papers of the RSA, vol. 56. Andersson, A.E., (1986), «The four logistical revolutions», Papers of the RSA, vol. 59.

Aydalot, P. (ed.), (1986), «Les milieux innovateurs en Europe», GREMI, Paris.

Castels, M., (1984), «Towards the information city ? High technology, economic change and spatial structure : some exploratory hypothesis», Institute of Urban and Regional Planning, WP, $\mathrm{n}^{\circ} 430$, University of California, Berkeley.

Crozier, M. et Friedberg, E., (1977), L'Acteur et le système, Seuil, Paris, p. 46.

De Bernardy, M. et Boisgontier, P., (1986), «Les micro-entreprises de la région grenobloise et leurs relations au tissu local», Table Ronde CER/ASDRLF, Aix-en-Provence, publié dans Planque (éd.), 1987.

Descles, J.P.,(1980), «Quelques systèmes de représentation linguistiques et métalinguistiques», CNRS ATP Analyse de Systèmes, Paris, p. 111.

Granie, P., (1985), «Politique régionale d'innovation et PMEi», thèse de doctorat, CER Aixen-Provence.

Lambooy, J.G., (1986), «Information and internationalization; dynamics of the relations of medium sized enterprises in a network environment», ASRDLF/CER Aix-en-Provence.

MacDonald, S., (1986), «Headhunting in high technology», Technovation, vol. 4, $\mathrm{n}^{\circ} 3$.

Maillat, D., (1987), «L'insertion des PME dans leur environnement local; quelques réflexions sur le cas des régions industrielles», Revue d'économie régionale et urbaine, numéro spécial, Planque (éd.).

Malecki, E.J., (1985), «Industrial location and corporate organization in high technology industries», Economic Geography, $n^{\circ} 4$. 
Merenne-Schoumaker, B., (1987), «Les centres d'entreprises», Revue d' économie régionale et urbaine, numéro spécial, Planque (éd.).

Perrin, J.C., (1985), «Redéploiement industriel et aménagement du territoire», Notes de Recherche du CER, Aix-en-Provence.

Perrin, J.C., (1987), «New technologies, local synergies, regional policies», GREMI, p. 7.

Planque, B., (1982), «Les Fonctions de recherche dans leur environnement local», Revue d'économie régionale et urbaine, $\mathrm{n}^{\circ} 3$.

Planque, B., (1983), Innovation et développement régional, Economica, Paris, chapitre III.

Planque, B.,(1985), «Le développement par les activités à haute technologie et ses répercussions spatiales. L'exemple de la Silicon Valley», Revue d'économie régionale et urbaine, $\mathrm{n}^{\circ} 5$.

Planque, B. et Py, B., (1987), «La dynamique de l'insertion des PME innovatrices dans leur environnement. Problématique et propositions méthodologiques», Revue d'économie régionale et urbaine, numéro spécial.

Planque, B., Py, B., Proulx, M. et Radjama, G., (1987), «La dynamique de l'insertion des PME innovatrices dans leur environnement», Cahiers du Centre d'économie régionale, $148 \mathrm{p}$. + annexes.

Planque, B. (éd.), (1987), «Les PME innovatrices dans leur environnement local etéconomique», numéro spécial de la Revue d'économie régionale et urbaine.

Porter, M.E., (1982), Choix stratégiques et concurrence, Economica, Paris.

Premus, R., (1982), «Location of high technology firms and regional economic development», Washington DC, US congress, Joint Economic Committee.

Radjama, G., (1986), «Pôles technologiques en Grande-Bretagne : le phénomène Cambridge», Notes de Recherche du CER, Aix-en-Provence.

Radjama, G., (1986), «Les activités à haute technologie en pays d'Aix. Étude préliminaire», CER, Aix-en-Provence.

Rogers, M., (1986), «The role of the research university in the spin-off of high technology companies», Technovation, vol. 04, $\mathrm{n}^{\circ} 3$.

Rogers, M. et Larsen, J., (1984), Silicon Valley Fever, Growth of High Technology Culture, Basic Books, New York.

Saxenian, A.L., (1981), «Silicon chips and spatial structure : the industrial basis of urbanisation in Santa Clara County, Calif.», Institute of Urban and Regional Planning, WP, $\mathrm{n}^{\circ} 435$, University of California Berkeley.

Segal, N., Quince, R., et associés (1985), «Cambridge phenomenon. The growth of high technology industry in a university town», Cambridge, RV.

Stohr, W.B., (1986), «Regional innovation complexes», Papers of the RSA, vol. 59.

Swyngedown, F. et Archer, K., (1985), «High technology development and regional space : same lessons from the American experience», Colloque «Technologies nouvelles : conditions de renouveau des régions en crise», Bruxelles. 